TITLE:

\title{
A note on the mirror-symmetric coherent structure in plane Couette flow
}

$\operatorname{AUTHOR}(\mathrm{S})$ :

Nagata, M.

\section{CITATION:}

Nagata, M.. A note on the mirror-symmetric coherent structure in plane Couette flow. Journal of Fluid Mechanics 2013, 727: R1.

ISSUE DATE:

2013-07

URL:

http://hdl.handle.net/2433/193965

RIGHT:

C2013 Cambridge University Press 


\title{
JFM RAPIDS journals.cambridge.org/rapids
}

\section{A note on the mirror-symmetric coherent structure in plane Couette flow}

\author{
M. Nagata $\dagger$ \\ Department of Aeronautics and Astronautics, Graduate School of Engineering, Kyoto University, \\ Kyoto 606-8501, Japan
}

(Received 18 March 2013; revised 23 April 2013; accepted 20 May 2013; first published online 14 June 2013)

We note that the mirror-symmetric solution in plane Couette flow, found recently by Gibson, Halcrow \& Cvitanović (J. Fluid Mech., vol. 611, 2009, pp. 107-130) and Itano \& Generalis (Phys. Rev. Lett., vol. 102, 2009, p. 114501), belongs to the solution group classified as 'ribbon' in rotating-plane Couette flow (RPCF). It represents a subcritical (in terms of the system rotation) solution at zero rotation rate on the three-dimensional tertiary flow branch which bifurcates from the second streamwise-independent flow in RPCF. The way of its appearance is similar to that of the Nagata solution (J. Fluid Mech., vol. 217, 1990, pp. 519-527), which lies on the subcritical three-dimensional tertiary flow branch bifurcating from the first streamwise-independent flow in RPCF.

Key words: bifurcation, nonlinear instability, transition to turbulence

\section{Introduction}

The problem of flow instabilities and transition to turbulence has recently attracted considerable attention. In particular, tremendous progress has been made in the past few decades for flows, such as plane Couette flow, pipe flow and square duct flow, where the basic states are linearly stable and, therefore, subcritical saddle-node bifurcations are the only possibility for non-trivial finite-amplitude solutions (see e.g. Kawahara, Uhlmann \& van Veen 2012, and references therein). These solutions are known as exact coherent structures (ECSs) and are believed to play an important role in transition to turbulence by constituting an invariant set (often figuratively called a skeleton) in the chaotic fluid motion. Such ECSs have been reported, for example, in plane Couette flow by Nagata (1990), Gibson, Halcrow \& Cvitanović (2009) and Itano 


\section{Nagata}

\& Generalis (2009), in pipe flow by Faisst \& Eckhardt (2003), Wedin \& Kerswell (2004) and Pringle \& Kerswell (2007), and in square duct flow by Wedin, Bottaro \& Nagata (2009), Okino et al. (2010), Uhlmann, Kawahara \& Pinelli (2010) and Okino \& Nagata (2012). Some of the ECSs in these three flows are characterized by a mirror-symmetric structure, which represents the highest degree of symmetry that the Navier-Stokes equations for wall-bounded shear flows possess.

In this short note we clarify the origin of the mirror-symmetric solution in plane Couette flow (Gibson et al. 2009; Itano \& Generalis 2009), in the framework of rotating-plane Couette flow (RPCF). To obtain ECSs in RPCF, we follow the method used by Wall \& Nagata (2006), that is, perturbations superimposed on the base flow are expanded by the Fourier series in the $x$ (streamwise) and $y$ (spanwise) directions and by the modified Chebyshev polynomials $T_{l}(z)$ in the $z$ (wall-normal) direction so as to satisfy the no-slip boundary conditions. A typical perturbation is expressed as

$$
q(x, y, z, t)=\sum_{l=-L}^{L} \sum_{m=-M}^{M} \sum_{n=-N}^{N} q_{l m n} \exp [\mathrm{i} m \alpha x+\mathrm{i} n \beta y] T_{l}(z),
$$

where $\alpha$ and $\beta$ are the wavenumbers in the streamwise and the spanwise directions, respectively. Upon discretizing the equations in the wall-normal direction using the collocation method, the resulting algebraic equations for the amplitudes, $q_{l m n}$, are solved by the Newton method.

\section{Basic equations}

The perturbed fluid motion from the basic state in a plane Couette system subject to spanwise rotation is governed, in the non-dimensional form, by the equation of continuity,

$$
\nabla \cdot \boldsymbol{u}=0
$$

and the equation of momentum conservation,

$$
\partial_{t} \boldsymbol{u}+\boldsymbol{U}_{B} \cdot \nabla \boldsymbol{u}+\boldsymbol{u} \cdot \nabla \boldsymbol{U}_{B}+\boldsymbol{u} \cdot \nabla \boldsymbol{u}+\bar{\Omega} \boldsymbol{j} \times \boldsymbol{u}=-\nabla p+\frac{1}{R e} \nabla^{2} \boldsymbol{u},
$$

where $\boldsymbol{u}=(u, v, w)$ is the velocity and $p$ is the pressure. The unit vectors in the streamwise, spanwise and wall-normal directions are denoted by $\boldsymbol{i}, \boldsymbol{j}$ and $\boldsymbol{k}$, respectively. The basic velocity is given by $\boldsymbol{U}_{B}(z)=-z \boldsymbol{i}$. The parameters that control the system are the Reynolds number, $R e$, and the rotation number, $\bar{\Omega}$, defined by

$$
R e=\frac{U_{0}^{*} d^{*}}{v^{*}}, \quad \bar{\Omega}=\frac{2 \Omega_{0}^{*} d^{*}}{U_{0}^{*}},
$$

respectively, where $\pm U_{0}^{*}$ is the velocity of the boundaries at $z^{*}=\mp d^{*}$, $\nu^{*}$ is the kinematic viscosity and $\Omega_{0}^{*}$ is the rotation rate, with the asterisk denoting a dimensional quantity.

In the case of a streamwise-independent flow, that is, $\partial_{x} \equiv 0, \boldsymbol{u}=(u, v, w)=$ $\left(u,-\partial_{z} \psi, \partial_{y} \psi\right),(2.2)$ simplifies to yield

$$
\begin{gathered}
\partial_{t} u+\left(v \partial_{y}+w \partial_{z}\right) u=(1-\bar{\Omega}) w+\frac{1}{\operatorname{Re} \Delta_{2} u,} \\
\partial_{t} \Delta_{2} \psi+J\left(\psi, \Delta_{2} \psi\right)=\bar{\Omega} \partial_{y} u+\frac{1}{\operatorname{Re}} \Delta_{2}^{2} \psi,
\end{gathered}
$$


Mirror-symmetric coherent structure in plane Couette flow

(a)

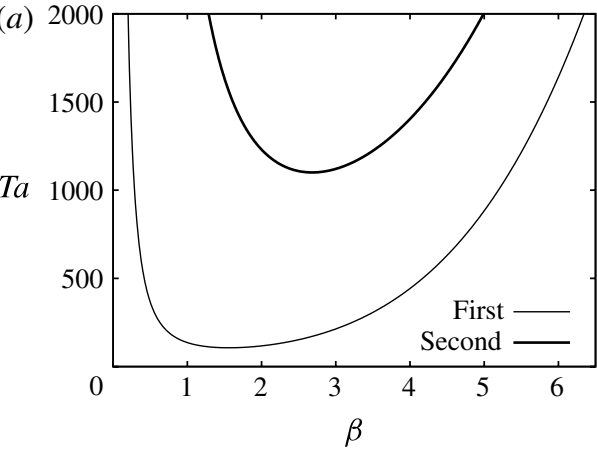

(b)

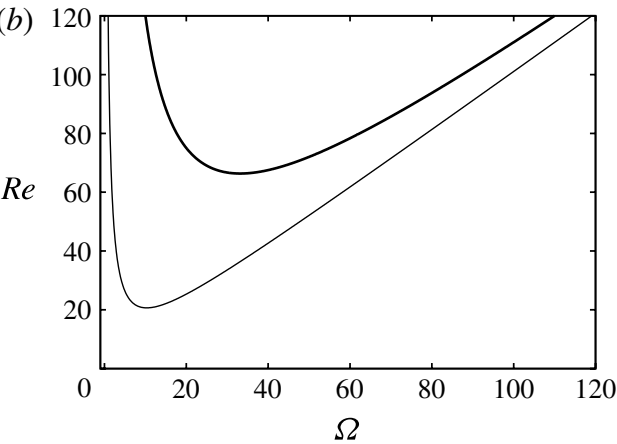

FIGURE 1. Neutral stability curves. The thin curve and the thick curve correspond to the first and the second linear critical states, respectively. $(a)$ The Taylor number, $T a$, versus the spanwise wavenumber, $\beta$. (b) The loci of $T a=T a_{c}^{(1)}=106.735$ and $T a=T a_{c}^{(2)}=1100.650$.

where $J(a, b)=\partial_{y} a \partial_{z} b-\partial_{z} a \partial_{y} b$ and $\Delta_{2}=\partial_{y}^{2}+\partial_{x}^{2}$. It can be shown that (2.4) and (2.5) are controlled by a single parameter called the Taylor number, $\mathrm{Ta}$,

$$
T a=\Omega(R e-\Omega),
$$

where $\Omega=\operatorname{Re} \bar{\Omega}$ (see the Appendix).

\section{Bifurcation analysis}

\subsection{The streamwise-independent flows}

It is well known that the basic laminar state of RPCF becomes unstable as Ta exceeds its critical value, $T a_{c}^{(1)}=106.735$, to a streamwise-independent perturbation with the spanwise wavenumber $\beta_{c}^{(1)}=1.5582$. For the purposes of the present problem, we note that the second critical state is reached at $T a_{c}^{(2)}=1100.650$ with $\beta_{c}^{(2)}=2.6823$, as shown in figure 1(a). The loci of $T a=T a_{c}^{(1)}$ and $T a=T a_{c}^{(2)}$ are traced in the $\Omega-R e$ plane in figure $1(b)$. Typical nonlinear streamwise-independent flows, referred to as Taylor vortex type I $\left(\mathrm{TV}_{1}\right)$ and Taylor vortex type II $\left(\mathrm{TV}_{2}\right)$, bifurcating from the first and the second critical states, respectively, are presented in figure 2. It can be seen, in particular, that $\mathrm{TV}_{2}$ is characterized by double-layered arrays of vortex rolls in comparison with the single-layered $\mathrm{TV}_{1}$. Both flows are mirror-symmetric about $y=0$ and the periodic boundaries $y= \pm \pi / \beta$.

\subsection{The three-dimensional mirror-symmetric solutions}

We find that branches of three-dimensional steady flows bifurcate from the first streamwise-independent flow $\mathrm{TV}_{1}$ at $\Omega=0.98$ and 2.82, and the second streamwiseindependent flow $\mathrm{TV}_{2}$ at $\Omega=8.32$ and 142.28, as shown in figure 3 . It is known that the three-dimensional wavy vortex flow (WVF) bifurcating from $\mathrm{TV}_{1}$ possesses the shift-rotation symmetry, $\Omega$,

$$
\boldsymbol{\Omega}:[u, v, w](x, y, z)=[-u, v,-w]\left(-x, y+\frac{\pi}{\beta},-z\right),
$$

and the shift-reflection symmetry, $\boldsymbol{S}$,

$$
S:[u, v, w](x, y, z)=[u,-v, w]\left(x+\frac{\pi}{\alpha},-y+\frac{\pi}{\beta}, z\right)
$$




\section{Nagata}

(a)

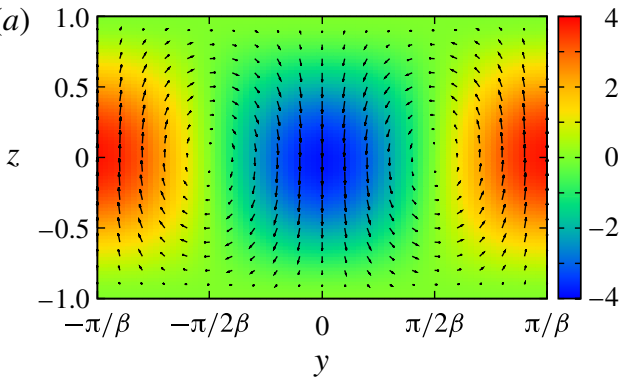

(b)

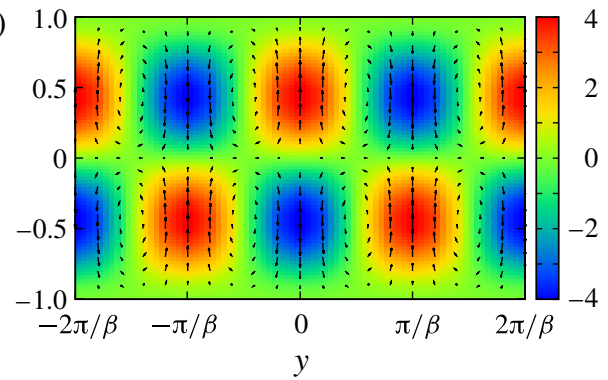

FIGURE 2. The cross-sectional flow structure of the streamwise-independent flows. (a) $\mathrm{TV}_{1}(-\pi / \beta \leqslant y \leqslant \pi / \beta)$ at $T a=150$ with $\beta=1.56$; and $(b) \mathrm{TV}_{2}(-2 \pi / \beta \leqslant y \leqslant 2 \pi / \beta)$ at $T a=1200$ with $\beta=2.68$. Arrows and contours represent the cross-sectional velocities, $(v, w)$, and the streamwise velocity, $u$, respectively. From the mathematical equivalence with the problem of two-dimensional Rayleigh-Bénard convection (see the Appendix), contours for $u$ can be regarded as those of temperature deviation, $\theta$, from the conductive state, with $T a$ being replaced by the Rayleigh number, $R a$. It can be seen that the wall-normal velocity component, $w$, is positive/negative in the regions for positive/negative $u$ or $\theta$ (i.e. locally hotter/colder regions) and that the streamwise vortices are accommodated between these regions.

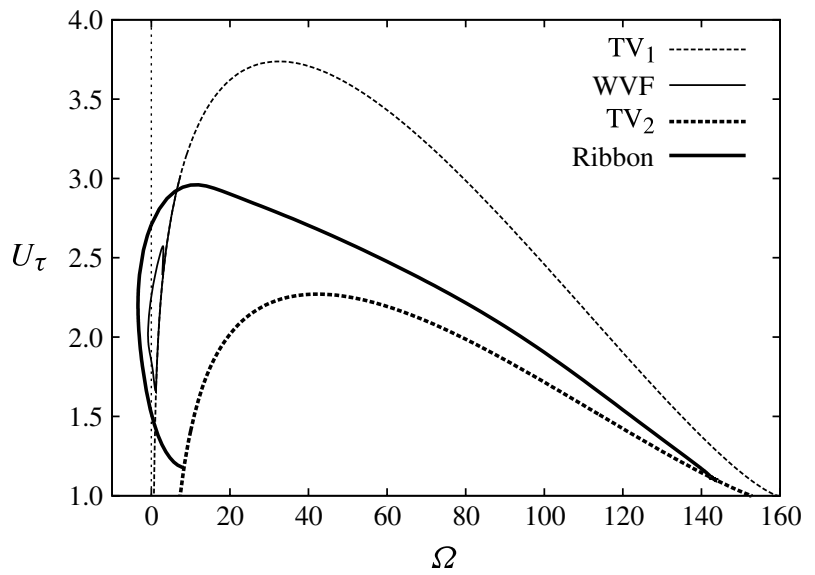

FIGURE 3. The momentum transport $U_{\tau}$ at the boundary $z=1$ for WVF and ribbon bifurcating from the first and the second streamwise-independent flows, $\mathrm{TV}_{1}$ and $\mathrm{TV}_{2}$, respectively. Parameters $R e=160, \alpha=0.9 ; \beta=1.5$ for $\mathrm{TV}_{1}$, WVF and ribbon, whereas $\beta=3.0$ for $\mathrm{TV}_{2}$.

and belongs to the solution class $\mathscr{A}_{2}$ classified in Nagata (1986). The plane of mirror symmetry at $y=0$ for $\mathrm{TV}_{1}$ is not carried over. The solution at $\Omega=0$ for $\mathscr{A}_{2}$ is often referred to as the Nagata solution in plane Couette flow (Nagata 1990).

It is found that the bifurcation from $\mathrm{TV}_{2}$ is subharmonic and that the resulting three-dimensional flow possesses the highest degree of symmetry possible in threedimensional RPCF, namely, the mirror symmetry, $\boldsymbol{Z}_{y}$, with respect to the plane $y=0$,

$$
\boldsymbol{Z}_{y}:[u, v, w](x, y, z)=[u,-v, w](x,-y, z),
$$

in addition to the symmetries, $\boldsymbol{\Omega}$ and $\boldsymbol{S}$, of $\mathscr{A}_{2}$. The solution class for this threedimensional mirror-symmetric flow is classified as $\mathscr{A}_{0}$ in Nagata (1986). The existence 


\section{Mirror-symmetric coherent structure in plane Couette flow}
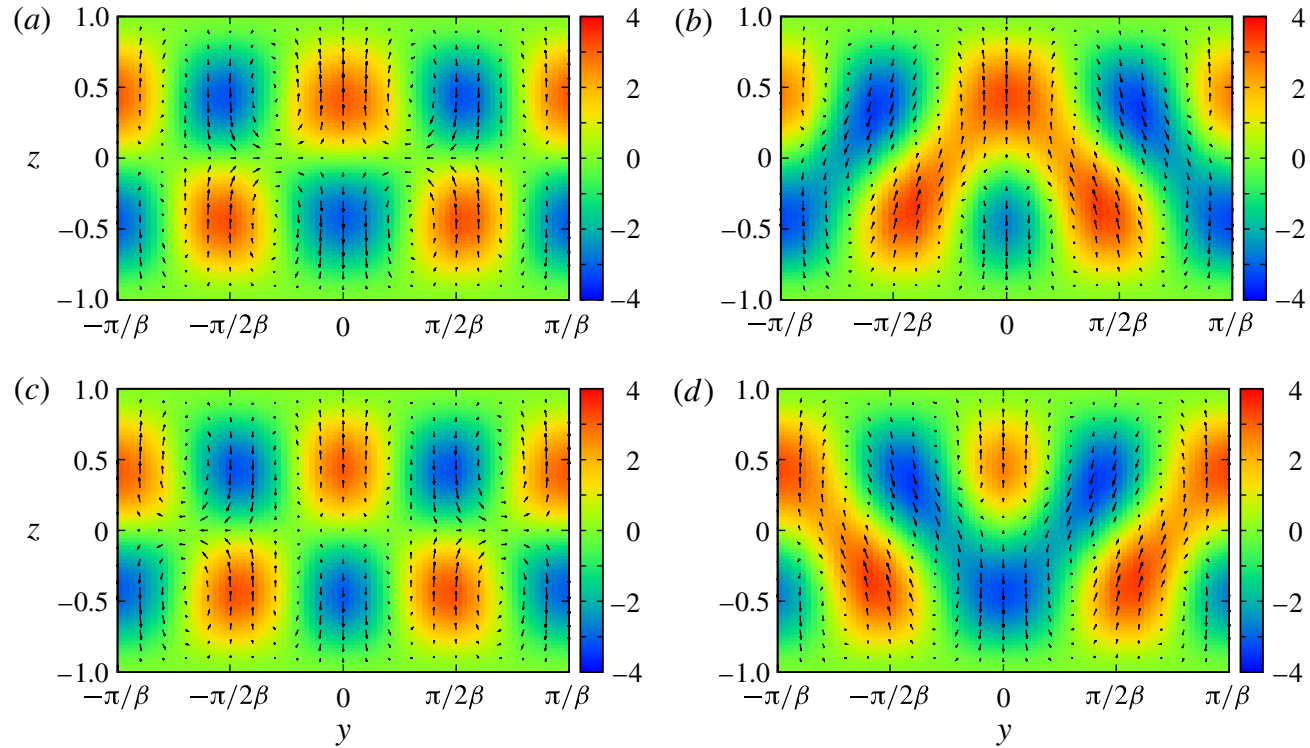

FIGURE 4. The cross-sectional flow field of the three-dimensional mirror-symmetric solution at $R=100$ and $\Omega=13$ with $(\alpha, \beta)=(0.8,1.56)$ at: $(a) x=0,(b) x=(1 / 4) 2 \pi / \alpha$, (c) $x=$ $(1 / 2) 2 \pi / \alpha$ and $(d) x=(3 / 4) 2 \pi / \alpha$. The spanwise period corresponds to twice the period of $\mathrm{TV}_{2}$, cf. figure $2(b)$. Arrows and contours represent the cross-sectional velocities, $(v, w)$, and the streamwise velocity, $u$, respectively. Note that the flow is mirror-symmetric about $y=0$ in addition to the periodic boundaries $y= \pm \pi / \beta$.

of the three-dimensional mirror-symmetric solution in RPCF has been reported in Nagata (1999), where the solution is referred to as 'ribbon', the terminology following that of the classification by Chossat \& Iooss (1994) for flows in the Taylor-Couette system.

The variation of the cross-sectional flow field along the streamwise direction for ribbon is presented in figure 4. Clearly, its double-layered structure is inherited from $\mathrm{TV}_{2}$. Regions of positive (or negative) $u$ in the upper and the lower layers merge, constituting a triad (figure $4 b$ ), and then split (figure $4 c$ ). They merge again, but with different triad components (figure $4 d$ ). Ribbon with such a triad formation would thus be a good candidate for the braided vortex flow observed in the circular Couette system with co-rotating cylinders considered by Andereck, Dickmann \& Swinney (1983). (Note that the circular Couette system in the narrow-gap limit between cylinders rotating with almost equal angular velocities is identical to RPCF (Nagata 1986).) The flow field in the $(x, y)$ plane corresponding to figure 4 is provided for $z=0.2$ in figure 5. The regions of high- and low-speed streaks on $z=0.2$ coincide with those of positive and negative $w$, respectively.

It can be seen in figure 3 that the subcritical branch of the mirror-symmetric solution for the class $\mathscr{A}_{0}$ also reaches the non-rotating case, $\Omega=0$. The mirrorsymmetric solutions at $\Omega=0$ correspond to the equilibrium solutions classified as $E Q_{7}$ and $E Q_{8}$ in Gibson et al. (2009) and the 'hairpin vortex state' of Itano \& Generalis (2009). The mirror-symmetric solution on the lower branch has been identified as an edge state at $R e=400$ in plane Couette flow (Duguet, Brandt \& Larsson 2010). The solutions at $\Omega=0$ for both classes correspond to the saddle-node bifurcations in plane 


\section{Nagata}

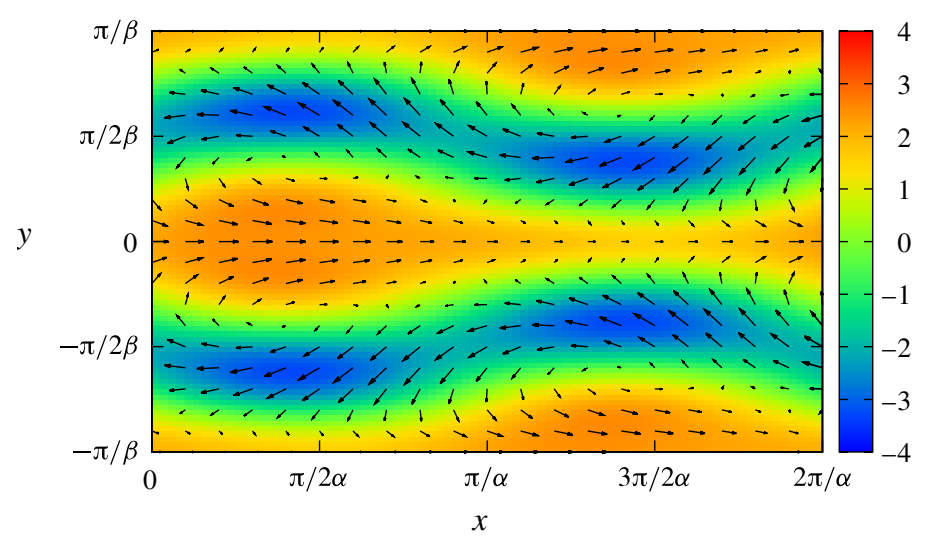

FIGURE 5. The flow structure of the mirror-symmetric solution in the $(x, y)$ plane at $z=0.2$. Parameter values are the same as those in figure 4. Arrows and contours represent the velocities, $(u, v)$, and the wall-normal velocity, $w$, respectively.

Couette flow. Our present calculations indicate that the minimum Reynolds number at the saddle node for the class $\mathscr{A}_{0}$ takes place at $R e=140.5$ with the wavenumber pair $(\alpha, \beta)=(0.76,1.37)$, while it is $\operatorname{Re}=127.7$ with $(\alpha, \beta)=(0.58,1.15)$ for the class $\mathscr{A}_{2}$. The flow structure of the mirror-symmetric solution in plane Couette flow can be found in Itano \& Generalis (2009) and Deguchi \& Nagata (2010).

\section{Summary}

The three-dimensional mirror-symmetric solution in plane Couette flow is found to lie on the branch of three-dimensional flows classified as ribbon, which bifurcate from points at which rotating-plane Couette flow loses stability to a second eigenmode that features a double-layered streamwise vortex structure. The solution itself is not new, but the present study that traces this solution on to RPCF can be valuable in analysing flows in the latter configuration, which have recently been studied experimentally by Tsukahara, Tillmark \& Alfredsson (2010). The recognition of two separate bifurcation routes originating from the first and the second eigenmodes would be important in understanding multiplicity and complexity of solutions in shear flow, in general (Mullin 2010).

We also add that a symmetry-breaking bifurcation of the class $\mathscr{A}_{2}$ from the branch of the class $\mathscr{A}_{0}$ has not been detected in plane Couette flow by our calculations for $R e$ up to 2000, although such a bifurcation should be possible since $\mathscr{A}_{2} \supset \mathscr{A}_{0}$.

Appendix. Mathematical equivalence between streamwise-independent flow in rotating-plane Couette flow and two-dimensional Rayleigh-Bénard convection

It has been known for several decades that there exists a theoretical analogy between rotating fluids and stratified fluids (Chandrasekhar 1961; Yih 1965). In particular, the analogy in turbulent states between curved shear flows and thermal flows (Bradshaw 1969) has been applied in describing turbulent Taylor-Couette flow and Rayleigh-Bénard convection (see e.g. Dubrulle \& Hersant 2002; Eckhardt, Grossmann \& Lohse 2007)). Moreover, a mathematical equivalence exists between problems of axisymmetric Taylor vortex flow in the narrow-gap limit of almost 


\section{Mirror-symmetric coherent structure in plane Couette flow}

co-rotating cylinders, or, alternately, streamwise-independent flow in rotating-plane Couette flow, and the two-dimensional Rayleigh-Bénard convection of a fluid with Prandtl number equal to unity (see e.g. Nagata 1986). This equivalence originates from the exact analogy for two-dimensional flows between rotating fluids and stratified fluids, which was first noted by Veronis (1970) and has also been used for obtaining a nonlinear solution in a convective flow with a constant shear by Clever \& Busse (1992). Therefore, the following deduction is not new, but it would be informative to restate here the mathematical equivalence in accord with our context.

By using the transformation $\left(\partial_{t}, u, v, w, \psi\right) \rightarrow(1 / \operatorname{Re})\left(\partial_{t}, \operatorname{Re}(1-\bar{\Omega}) u, v, w, \psi\right)$ and $\Omega=\operatorname{Re} \bar{\Omega}$, one can find that equations (2.4) and (2.5) for streamwise-independent flows in RPCF reduce to

$$
\begin{gathered}
\partial_{t} u+\left(v \partial_{y}+w \partial_{z}\right) u=w+\Delta_{2} u, \\
\partial_{t} \Delta_{2} \psi+J\left(\psi, \Delta_{2} \psi\right)=\Omega(\operatorname{Re}-\Omega) \partial_{y} u+\Delta_{2}^{2} \psi .
\end{gathered}
$$

Thus, the streamwise-independent flow is controlled by a single parameter, $\mathrm{Ta}$, defined in expression (2.6).

For Rayleigh-Bénard convection of a horizontal fluid layer, perturbations $\boldsymbol{u}, \theta$ from the conductive state with the temperature profile, $\Theta(z)=-z$, are governed, in non-dimensional form, by

$$
\begin{gathered}
\partial_{t} \theta+\boldsymbol{u} \cdot \nabla \theta=w+\nabla^{2} \theta, \\
\partial_{t} \boldsymbol{u}+\boldsymbol{u} \cdot \nabla \boldsymbol{u}=-\nabla p+\operatorname{Ra} \operatorname{Pr} \theta \boldsymbol{k}+\operatorname{Pr} \nabla^{2} \boldsymbol{u} .
\end{gathered}
$$

The parameters that control the system are the Rayleigh number, $R a$, and the Prandtl number, $\operatorname{Pr}$, defined by

$$
R a=\frac{g^{*} \gamma^{*} \Delta T^{*} d^{* 3}}{\nu^{*} \kappa^{*}}, \quad \operatorname{Pr}=\frac{\nu^{*}}{\kappa^{*}},
$$

respectively, where $g^{*}$ is the acceleration due to gravity, $\gamma^{*}$ is the volume expansion coefficient, $\kappa^{*}$ is the thermal diffusivity and $\Delta T^{*}$ is the temperature difference between the top and the bottom boundaries separated by the distance $2 d^{*}$.

In the case of two-dimensional convection rolls (where $\partial_{x} \equiv 0, \boldsymbol{u}=(0, v, w)=$ $\left.\left(0,-\partial_{z} \psi, \partial_{y} \psi\right)\right)$, equations (A 4) and (A 3) simplify to yield

$$
\begin{gathered}
\partial_{t} \theta+\left(v \partial_{y}+w \partial_{z}\right) \theta=w+\Delta^{2} \theta, \\
\partial_{t} \Delta_{2} \psi+J\left(\psi, \Delta_{2} \psi\right)=\operatorname{Pr}\left(\operatorname{Ra} \partial_{y} \theta+\Delta_{2}^{2} \psi\right) .
\end{gathered}
$$

Thus, the mathematical equivalence between system (A 1), (A 2) and system (A 6), (A 7) holds, with $\theta=u$ and $R a=\Omega(R e-\Omega)=T a$ for $\operatorname{Pr}=1$.

\section{References}

Andereck, C. D., Dickmann, R. \& Swinney, H. 1983 New flows in a circular Couette system with co-rotating cylinders. Phys. Fluids 26, 1395-1401.

BRADSHAW, P. 1969 The analogy between streamline curvature and buoyancy in turbulent shear flow. J. Fluid Mech. 36, 117-191.

CHANDraseKHAR, S. 1961 Hydrodynamic and Hydromagnetic Stability. Oxford University Press.

Chossat, P \& Iooss, G 1994 The Couette-Taylor Problem, Applied Mathematical Sciences, vol. 102. Springer.

Clever, R. M. \& Busse, F. H. 1992 Three-dimensional convection in a horizontal fluid layer subject to a constant shear. J. Fluid Mech. 234, 511-527. 


\section{Nagata}

Deguchi, K. \& Nagata, M. 2010 Travelling hairpin-shaped fluid vortices in plane Couette flow. Phys. Rev. E 82, 056325.

Dubrulle, B. \& Hersant, F. 2002 Momentum transport and torque scaling in Taylor-Couette flow from an analogy with turbulent convection. Eur. Phys. J. B 26, 379-386.

Duguet, Y., BRAndT, L. \& LARSSON, B. R. 2010 Towards minimal perturbations in transitional plane Couette flow. Phys. Rev. E 82, 026316.

Eckhardt, B., Grossmann, S. \& Lohse, D. 2007 Torque scaling in turbulent Taylor-Couette flow between independently rotating cylinders. J. Fluid Mech. 581, 221-250.

Faisst, H. \& Eckhardt, B. 2003 Traveling waves in pipe flow. Phys. Rev. Lett. 91, 224502.

Gibson, J. F., Halcrow, J. \& Cvitanović, P. 2009 Visualizing the geometry of state space in plane Couette flow. J. Fluid Mech. 611, 107-130.

ItAno, T. \& Generalis, S. C. 2009 Hairpin vortex solution in planar Couette flow: a tapestry of knotted vortices. Phys. Rev. Lett. 102, 114501.

Kawahara, G., Uhlmann, M. \& Van Veen, L. 2012 The significance of simple invariant solutions in turbulent flows. Annu. Rev. Fluid Mech. 44, 203-225.

Mullin, T. 2010 The rich structure of transition in a shear flow. J. Fluid Mech. 684, 1-4.

NagATA, M. 1986 Bifurcations in Couette flow between almost corotating cylinders. J. Fluid Mech. 169, 229-250.

NAGATA, M. 1990 Three-dimensional finite-amplitude solutions in plane Couette flow: bifurcation from infinity. J. Fluid Mech. 217, 519-527.

Nagata, M. 1999 Ribbons in rotating plane Couette system. In Proceedings of 11th International Couette-Taylor Workshop (ed. C. Egbers \& G. Pfister), pp. 67-68. Bremen, Germany.

OKInO, S. \& NAGATA, M. 2012 Asymmetric travelling waves in a square duct. J. Fluid Mech. 693, $57-68$.

Okino, S., Nagata, M., Wedin, H. \& Bottaro, A. 2010 A new nonlinear vortex state in square-duct flow. J. Fluid Mech. 657, 413-429.

Pringle, C. C. T., Duguet, Y. \& Kerswell, R. R. 2009 Highly symmetric travelling waves in pipe flow. Phil. Trans. R. Soc. A 367, 457-472.

Pringle, C. C. T. \& Kerswell, R. R. 2007 Asymmetric, helical and mirror-symmetric travelling waves in pipe flow. Phys. Rev. Lett. 99, 074502.

Tsukahara, T., Tillmark, N. \& Alfredsson, P. H. 2010 Flow regimes in a plane Couette flow with system rotation. J. Fluid Mech. 648, 5-33.

Uhlmann, M., Kawahara, G. \& Pinelli, A. 2010 Travelling waves consistent with turbulence-driven secondary flow in a square duct. Phys. Fluids 22, 084102.

Veronis, G. 1970 The analogy between rotating and stratified fluids. Annu. Rev. Fluid Mech. 2, 37-66.

WALl, D. P. \& NaGATA, M. 2006 Nonlinear secondary flow through a rotating channel. J. Fluid Mech. 564, 25-55.

Wedin, H., Bottaro, A. \& Nagata, M. 2009 Three-dimensional traveling waves in a square duct. Phys. Rev. E 79, 065305.

WEDIN, H. \& KERSWELL, R. R. 2004 Exact coherent structures in pipe flow: travelling wave solutions. J. Fluid Mech. 508, 333-371.

YIH, C.-S. 1965 Dynamics of Nonhomogeneous Fluids. Macmillan. 